\title{
Development and Evaluation of a Fluidic Facemask for COVID-19 Transmission Mitigation
}

\author{
David Keisar $\mathrm{MSc}^{1 \dagger}$, Anan Garzozi MSc ${ }^{1 \dagger}$, Prof. Moshe Shoham $\mathrm{PhD}^{2}$, and Prof. David Greenblatt $\mathrm{PhD}^{2 *}$ \\ ${ }^{1}$ Grand Technion Energy Program (GTEP), Technion - Israel Institute of Technology, Technion Campus, \\ Haifa 3200003, Israel \\ ${ }^{2}$ Faculty of Mechanical Engineering, Technion - Israel Institute of Technology, Technion Campus, Haifa \\ 3200003, Israel
}

*Corresponding author: davidg@technion.ac.il. https://orcid.org/0000-0002-0789-5690

$†$ The author equally contributed

Received:----; Revised:----; Accepted:-----;

Keywords: weakly advected turbulent jets; COVID-19; personal protective equipment; Air-Screen; Air curtain; Fluidic face mask.

Abstract

A novel fluidic facemask, or Air-Screen, is described and demonstrated experimentally for protection against virus-laden aerosol and droplet infections, such as COVID-19. The Air-Screen employs a small, high-aspect-ratio, crossflow fan mounted on the visor of a filter-covered cap to produce a rectangular air jet, or screen, in front of the wearer's face. A theoretical model, based on a weekly-advected jet in a crossflow, indicated its effectiveness against a wide spectrum of virus-laden aerosols and droplets. Qualitative flow visualization experiments, using a mannequin, clearly illustrated the AirScreen's ability to effectively block airborne droplets $\left(\sim 10^{0} \mu \mathrm{m}\right)$ from the wearer's face. Quantitative experiments to simulate droplets produced during sneezing or a wet cough $\left(\sim 10^{2} \mu \mathrm{m}\right)$, were propelled (via a transmitter) at an average velocity of $50 \mathrm{~m} / \mathrm{s}$ at $1 \mathrm{~m}$ from the mannequin or a target. The Air-Screen blocked $62 \%$ of all droplets with a diameter of less than $150 \mu \mathrm{m}$. With an Air-Screen active on the transmitter, 99\% of all droplets were blocked and when both mannequin and transmitter Air-Screens were active, $99.8 \%$ all droplets were blocked. In contrast to conventional facemasks, the AirScreen is remarkably effective in protecting the wearer and others from an infected wearer.

Impact Statement

The physical principle underpinning the Air-Screen's operation is that drag forces, imposed on virus-laden airborne droplets by a nominally two-dimensional turbulent jet, deflects them downwards, away from the face of the wearer. The simplest valid theoretical model is that of a two-dimensional jet interacting with a droplet-laden crossflow, intended to model anticipated movements of the wearer or close-range oral communication. A mathematical model for weakly advected turbulent jets is adopted, including the crossflow effects on the spreading rate and the jet trajectory. This model facilitated the optimum geometric positioning and orientation of the jet for the purpose of effectively blocking droplets less than $100 \mu \mathrm{m}$. The excellent correspondence between the mathematical model and experiments is a clear translational demonstration of fluid mechanics principles applied to the mitigation of the COVID-19 virus in particular, and airborne respiratory viruses in general. 


\section{Introduction}

The most pervasive and visible consequence of the recent COVID-19 pandemic is the wearing of surgical or cotton facemasks for the purpose of mitigating human to human transmission of the virus. The U.S. Centers for Disease Control and Prevention (CDC) determined that an infected or asymptomatic individual wearing a surgical mask protects others from the wearer (CDC, 2021). This is because the primary mechanism of transmission between individuals is considered to be virus-laden aerosols and droplets (Bourouiba, Dehandschoewercker, \& Bush, 2014). However, it is still unclear how effective masks are in protecting the wearer from being infected by others (Teesing, Straten, Man, \& Horeman-franse, 2020), and the U.S. Food and Drug Administration (FDA) has not approved any surgical mask specifically for protection against the coronavirus (Laine, Goodman, \& Guallar, 2020). Moreover, even when wearing a surgical mask, isolated droplets can still be spread beyond $70 \mathrm{~cm}$ (Dbouk \& Drikakis, 2020b) or even greater distances when using bandanas or N-95 masks (Arumuru, Pasa, \& Samantaray, 2020; Verma, Dhanak, \& Frankenfield, 2020). Likewise, in the presence of wind towards the wearer, as in open-air environments, large droplets (up to $80 \mu \mathrm{m}$ ) can be carried up to 6 meters downwind (Dbouk \& Drikakis, 2020a). The distinction between aerosols and droplets, traditionally considered to be less than $5 \mu \mathrm{m}$ to $10 \mu \mathrm{m}$, has important implications for transmission. For example, $10 \mu \mathrm{m}$ aerosols remain airborne for approximately 500 seconds in the absence of evaporation and are sometimes denoted "airborne droplets" (Bhagat, Wykes, Dalziel, \& Linden, 2020). A detailed review, recently published in Science by Wang et al. asserts that virus-laden aerosols between $5-100 \mu \mathrm{m}$ are responsible for transmission, while larger "droplets" (>100 $\mu \mathrm{m})$ travel less than a meter and fall to the ground within seconds to produce fomites (contaminated surfaces).

The effectiveness of surgical and other facemasks is usually determined for virus-laden droplets with diameters greater than $D=5 \mu \mathrm{m}$ (Fennelly, 2020), usually generated by sneezing and coughing. However, smaller-sized virus-laden aerosols with diameters less than $5 \mu \mathrm{m}$, (Bakhit et al., 2021; Fennelly, 2020) are considered a primary transmission source for other viral diseases, such as SARS-Cov-2. These aerosols can be carried indefinitely (Laine et al., 2020) and are products of all air exhalation such as oral communication, expiration, sneezing and coughing. Most commercial off-the-shelf facemasks are unable to filter aerosols smaller than $5 \mu \mathrm{m}$ effectively. In fact, even aerosols smaller than $1 \mu \mathrm{m}$ are not effectually filtered by standard approved (ASTM F1862 / F1862M-17, 2017; BS EN 14683, 2019) IIR surgical masks. A detailed review (Wang et al., 2021), asserts that virus-laden aerosols between 5-100 $\mu \mathrm{m}$ are responsible for transmission, while larger "droplets" $(>100 \mu \mathrm{m})$ travel less than a meter and fall to the ground within seconds to produce fomites (contaminated surfaces).

Many individuals find the wearing of masks to an irritant or even highly uncomfortable. Masks unquestionably inhibit facial recognition and verbal communication, and some people experience a "psychological" difficulty of breathing (Bakhit et al., 2021). There also appears to be some controversy whether face masks may increase rebreathing of $\mathrm{CO}_{2}$ in children (Kisielinski et al., 2021). Transparent face shields are touted as a potential alternative because of the physical barrier without covering the mouth and nose. However, virus-laden aerosolized particles can escape below the face shield (Arumuru et al., 2020). Furthermore, they are ungainly and tend to become contaminated with exhaled droplets or due to contact with unsterilized surfaces after a short use (Kumar et al., 2020). There is, clearly, an urgent need for a comfortable facemask, does not inhibit breathing and has no negative impact on facial recognition or verbal communication. This article discusses the development and evaluation of such a facemask.

Recently, a fluidic facemask, or 'Air Curtain' concept, was proposed by Sakharov and Zhukov (2020), involving a rectangular jet of air blown in front of the face. The concept is similar to the well-known and effective concept of an air curtain or "fluidic barrier" at the doorway of a ventilated building (Frank \& Linden, 2014), and the concept is often employed in operating theaters (Barbadoro, 2016). A significant advantage is that it serves to protect both wearers from being infected and/or infecting when worn by two individuals. Although the concept has merit, the analysis performed in (Sakharov \& Zhukov, 2020) has several weaknesses. First, the issuing jet is assumed to have a constant width and speed, which is not physically realistic because both laminar and turbulent jets spread to conserve momentum. Second, their analysis does not account for the deflection of the jet due to the relative motion between the jet and the surroundings.

In order to evaluate the concept, we built a fluidic facemask, which embodies a practical and portable implementation of the fluidic barrier by integrating a small fan and power supply into a cap (see Figure 1). The outer, visible part of 
the cap (Figure 1, left), comprises a flexible frame that is covered with an antibacterial, porous material to minimize the pressure drop, and one or more high-efficiency particulate air (HEPA) filters (Contal et al., 1999; Zhang, Deng, Wang, \& Lin, 2020) or other high-quality standard mask filters (Teesing et al., 2020). The inner part of the cap is made from a comfortable, high-pressure-drop material (Figure 1, right). The rim is sealed, and there is a gap between the inner and outer parts of the cap that facilitates airflow with minimal pressure losses. The Air-Screen is produced by a crossflow fan mounted in the visor, together with an inlet guide vane (IGV) to maximize its efficiency (see Figure 1, right). At 1.2 watts, the fan produces $30 \mathrm{~Pa}$ of suction pressure and has a maximum flow rate of 5.12 liter/s, corresponding to an exit velocity of $10.2 \mathrm{~m} / \mathrm{s}$. Crossflow fans are ideal for this application because they produce a good balance between pressure and volumetric flow rate, with average specific speeds (Dang \& Bushnell, 2009). In other words, crossflow fans can suck air through filters (overcoming the pressure loss) and deliver an effective fluidic barrier jet in front of the face. Moreover, crossflow fans have the ability to produce a rectangular jet and produce low noise due to the structure made of segments of staggered forward-facing blades (Dang \& Bushnell, 2009). Also mounted on the cap visor is a battery charger, an on/off switch and voltage conversion electronics.

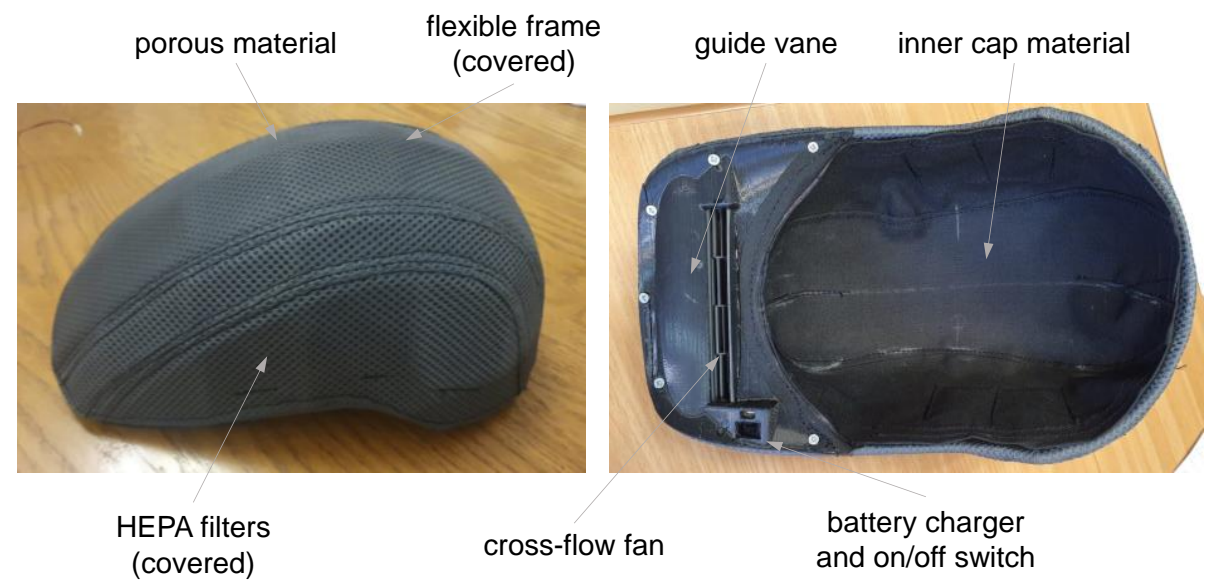

Figure 1. Two views of the Air-Screen: left - external visible top view of the cap; right - bottom view of the cap.

The global objective of this study is to evaluate the effectiveness of the Air-Screen concept, specifically applied to the design described above. A numerical model was developed to assess the viability of the concept, and experiments were performed to qualify and quantify the effectiveness of the Air-Screen to protect wearers both from small-sized airborne aerosols $\left(D \sim 10^{\circ} \mu \mathrm{m}\right)$ produced during breathing and verbal communication and large droplets $\left(D \sim 10^{2} \mu \mathrm{m}\right)$ produced by sneezing and hard coughing (Bourouiba et al., 2014; Scharfman, Techet, Bush, \& Bourouiba, 2016). In all cases, the assumption is that social distancing is not enforced between individuals. The paper is structured as follows: A physical model is developed, and simulations are performed that serve as a theoretical basis for the research. The prototype is described in section 2; different experimental setups are described in section 3 , and the main results with discussion are presented in section 4. Conclusions, with recommendations for future evaluations and research, are presented in section 5 .

\section{Physical Model Development}

The objective of the physical model was to predict the aerosol and droplet trajectories when encountering the AirScreen jet. Two main limitations, presented in (Sakharov \& Zhukov, 2020), were addressed. First, the constant jet width assumption was replaced by that of a turbulent jet (Schlichting \& Gersten, 2016), modified by a weak crossflow (Huang, Davidson, \& Nokes, 2005). Second, realistic particle sizes and speeds, covering the entire spectrum of virusladen aerosols and droplets, with representative Reynolds numbers, were implemented. Sections 2.1 to 2.2 describe the theory of two-dimensional (2-D) turbulent jets in a crossflow and the forces acting on droplets and aerosols, and section 2.3 presents the results of the numerical simulations. 


\subsection{Turbulent Jet-in-a-Crossflow}

The theoretical development of 2-D turbulent jets relies on conservation of momentum (Schlichting \& Gersten, 2016), together with the solution of the boundary layer equations, resulting in the velocity component in the streaming direction $(x)$ :

$$
u=u_{\mathrm{c}} \operatorname{sech}^{2}(\eta)
$$

and the crossflow direction $(y)$ :

$$
v=u_{\mathrm{c}}\left(2 \eta \operatorname{sech}^{2} \eta-\tanh \eta\right) / 2 \sigma
$$

where $\eta=\sigma(y / x), u_{\mathrm{c}} \sim\left(x-x_{0}\right)^{-1 / 2}$ is the centerline velocity, and $\sigma$ and $x_{0}$ are empirical constants (see definitions in Figure 2). The interaction of the fluidic barrier with virus-laden aerosols and droplets can be modeled as: (a) the interaction between rigid bodies (droplets) and a fluidic jet; (b) the interaction between a fluidic jet and a virus-laden crossflow; or (c) a combination of the two. Dbouk \& Drikakis (2020a) show that droplets and aerosols produced during a strong cough cannot travel more than $2 \mathrm{~m}$ in distance, where are the vast majority will not be carried more than $1 \mathrm{~m}$, and mostly spread via airborne transport. This correlates to the study of Wang et al. (2021) that estimates that only a small percentage of infection is carried via large droplet infection. Thus, a more accurate method to model virus-laden and Air-Screen interaction is between a fluidic jet and an airborne virus-laden crossflow.

Three-dimensional and two-dimensional turbulent jets in crossflows were studied extensively (Cárdenas, Suntz, Denev, \& Bockhorn, 2007; Gutmark \& Wygnanski, 1976; Haniu \& Ramaprian, 1989; Huang et al., 2005; Kamotani \& Greberf, 1972; Thompson, 1965). Huang et al. (2005) classify 2-D jets in a crossflow into two main categories, namely 2-D discharges in weak or strong crossflow. Irrespective of the crossflow strength, the jet is deflected due to two main mechanisms, namely a momentum balance between the jets and a pressure gradient across the jet that is expressed as a "drag" force. Thus, the trajectory of the jet's centerline can be expressed as:

$$
\frac{d y}{d x}=\frac{U_{a}\left(Q-Q_{0}\right)+\left(C_{d} / 2\right) U_{a}^{2} x}{U_{0}^{2} h}
$$

where $U_{a}$ is the crossflow velocity, $\left(Q-Q_{0}\right)$ is the difference between slot and local downstream volume flux, $C_{d}$ is the drag coefficient and $h$ is the slot width. However, a turbulent jet discharging into a very weak crossflow resembles a turbulent jet discharging into an ambient fluid (see Figure 2b). For these weakly advected jets, it can be assumed that the only interaction between the flows is a momentum transfer which is valid under the assumption (Huang et al., 2005):

$$
\frac{x}{U_{0}^{2} h / U_{a}^{2}} \leq 0.4
$$

and for these flows, we can assume that $C_{d}=0.8$ (Huang et al., 2005). 
(a)

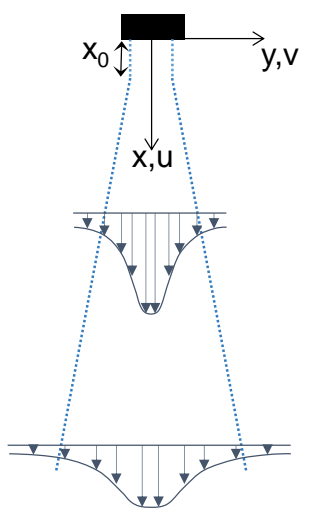

(b)

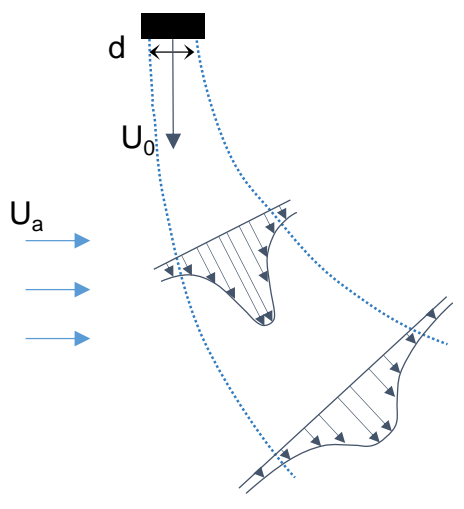

Figure 2. (a) Schematic of a two-dimensional turbulent jet; (b) schematic of a 2-D weakly advected turbulent jet in a crossflow.

These approximations allow an initial sizing of the crossflow fan selected for this study (see section 1). To obtain $U_{0}=9 \mathrm{~m} / \mathrm{s}$, a 1.2 watt fan was selected with an impeller length and diameter of 100 and $20 \mathrm{~mm}$, respectively. The jet exit slot was $h=5 \mathrm{~mm}$, resulted in a jet aspect ratio of 20 and is therefore assumed to be effectively two-dimensional. Although the input power can be regulated, all experiments were performed under nominal (1.2 watts) conditions. Several velocity profiles downstream of the jet exit were surveyed with a small flattened pitot tube in conjunction with a Lambrecht micro-manometer with a $\pm 700 \mathrm{~Pa}$ pressure range and a $\pm 0.2 \mathrm{~Pa}$ resolution. An example of two velocity profiles, together with the theoretical results (see eqn. (1)), are shown in Figure 3 and indicate a close correspondence based on the jet spreading rate constant $\sigma=7.67$.

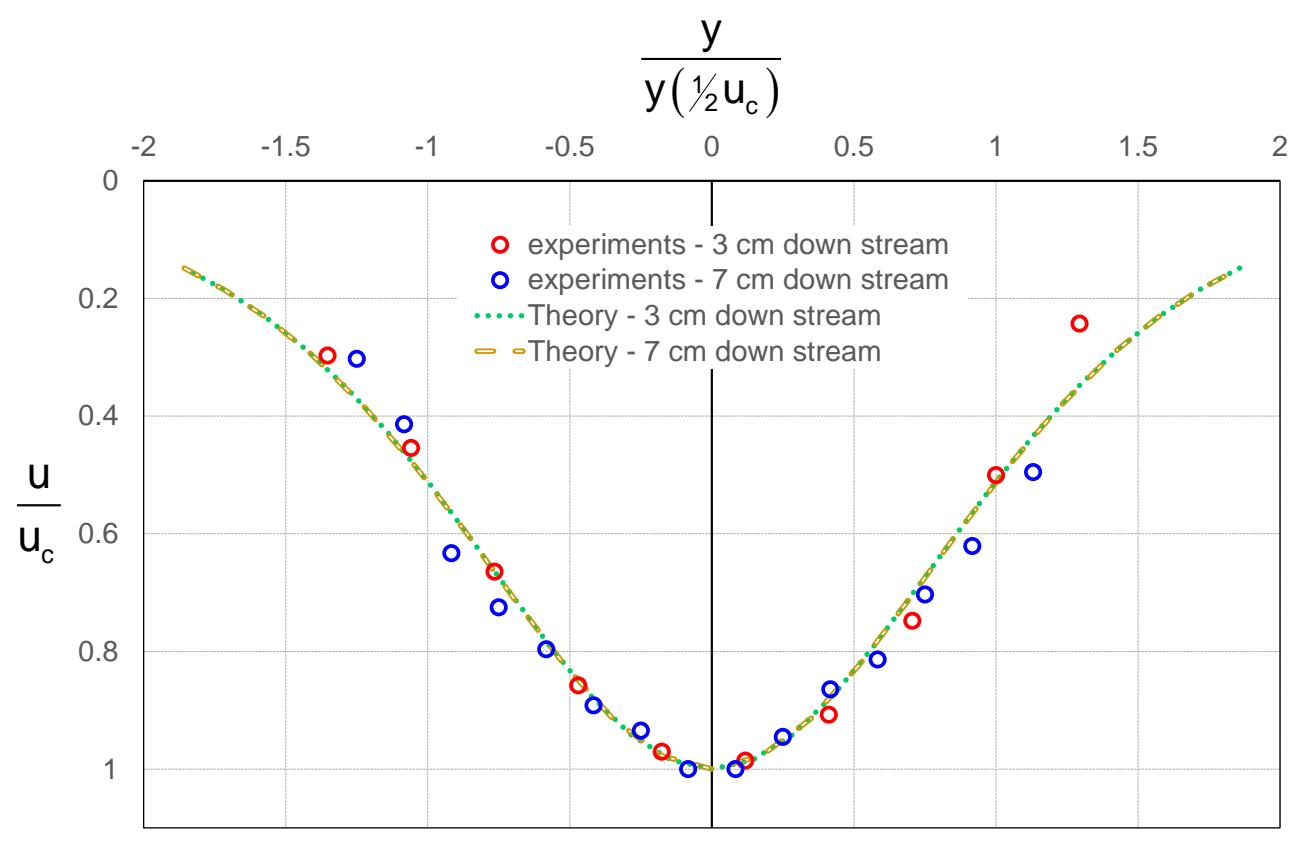

Figure 3. Non-dimensionalised turbulent velocity profiles measured at $3 \mathrm{~cm}$ and $7 \mathrm{~cm}$ downstream of the fan exit plane, shown together with the theoretical result shown in eqn. (1).

\subsection{Forces Acting on Aerosols and Droplets}

The range of virus-laden aerosol and droplet diameters varies between $\mathcal{O}\left(10^{\circ}\right)$ to $\mathcal{O}\left(10^{2}\right) \mu \mathrm{m}$, and this results in a relatively large Reynolds numbers range $\left(\operatorname{Re} \leq 10^{2}\right)$ where: 


$$
R e \equiv \frac{\left|\vec{u}_{\mathrm{rel}, \mathrm{drop}}\right| D}{v_{a}}
$$

$\left|\vec{u}_{\text {reld,rop }}\right|$ is the relative velocity magnitude between the aerosol velocity vector $\vec{u}_{\text {drop }}$ and the surrounding flow $\vec{U}$ and $v_{a}$ is the air kinematic viscosity. This results in more than an order of magnitude variation in the aerosols or droplet drag coefficient $C_{D \text {,drop }}$ (Proudman \& Pearson, 1957). To account for the large Reynolds number variation, the approximation attributed to Brown et al. (Brown \& Lawler, 2003) was used, namely:

$$
C_{D}=\frac{24}{R e}\left(1+0.15 R e^{0.681}\right)+\frac{0.407}{1+8710 / R e}
$$

which is valid for $R e \leq 10^{2}$. In order to compute the drag force, the relation:

$$
\left|\vec{F}_{D}\right|=-\frac{\pi}{8} C_{D} D^{2} \rho_{a}\left|\vec{u}_{\text {rel,drop }}\right|^{2}
$$

was used, where $\rho_{a}$ is the air density. In addition, forces due to gravity and buoyancy were calculated according to:

$$
\vec{F}_{b}=\frac{\pi}{6}\left(\rho_{a}-\rho_{f}\right) \vec{g} D^{3} \approx-\frac{\pi}{6} \rho_{f} g D^{3} \cdot \hat{x}
$$

where $\rho_{f}$ is the density of the aerosols and droplets (Sarkar, Xu, \& Lee, 2019).

\subsection{Numerical Model and Results}

At the outset, the combined velocity field of the jet and crosswind was solved numerically across the entire domain, as explained in section 2.1. Droplet size, location and velocity boundary conditions (Cauchy boundary conditions) were selected. Thus, the initial conditions $(t=0)$ for the simulations were the weakly advected jet velocity field together with the specific droplet parameters and boundary conditions. An explicit $4^{\text {th }}$ order Runge-Kutta method (Kincaid \& Cheney, 2002) was used to approximate the aerosol or particle locations at each time step, $\Delta t$, based on the current values and a weighted average of four increments. For each increment, based on the droplet's location and its prior velocity vector, the relative velocity vector was determined, thereby facilitating the calculation of $\operatorname{Re}$ and hence $C_{D}$. On the basis of these results, the droplet acceleration vector was estimated, and its location at $t+\Delta t$ was calculated. The accumulated error of the model is on the order of $(\Delta t)^{4}$; thus, the implementation of small $\Delta t$ ensured that the numerical errors are secondary compared to the model assumption errors.

Although the jet parameters were defined in the previous section, the Air-Screen retains several degrees of freedom, in particular (a) the slot distance from the face $y_{\text {jet }}$ and (b) the jet angle with respect to the vertical $\alpha_{\text {jet }}$ (see schematic in Figure 4, right). The simplest geometric configuration is to direct the jet vertically downwards $\left(\alpha_{\text {jet }}=0^{\circ}\right)$, as is done with air-curtains for ventilation control (Frank \& Linden, 2014). For the Air-Screen application, however, increasing the jet angle towards the horizontal away from the face $\left(\alpha_{\text {jet }}>0^{\circ}\right)$ increases its effectiveness as a fluidic barrier. Additionally, increasing the jet distance between the fan slot and face $\left(y_{\text {jet }}\right)$ also reduces interactions between the jet and face. We can therefore argue intuitively that the combined increase in both $\alpha_{\text {jet }}$ and $y_{\text {jet }}$ will bring about the greatest effect.

The numerical results are summarized as a contour plot in Figure 4 (left), which identifies the maximum airborne droplet diameter effectively blocked by the jet as a function of $y_{\text {jet }}$ and $\alpha_{\text {jet }}$, where body forces are neglected $\left(\vec{F}_{b}=0\right.$

). Neglecting body forces produces conservative results because when they are included, gravity dominates over buoyancy and their net effect is downwards. Figure 4 (right) also graphically identifies the relevant coordinates and 
parameters. Droplets are considered effectively "blocked" when their penetration of the jet falls below $15 \mathrm{~cm}$ from the jet slot height. The distance of $15 \mathrm{~cm}$ is based on the average of male and female anthropometric measurements between the mid-forehead and base of the chin, encompassing many different countries and ethnic groups (Farkas et al., 2005). The bright area in the lower left-hand part of the contour plot indicates poor effectiveness against airborne aerosols and droplets, which penetrate across the jet. Relatively large increases in either of these parameters, e.g. $y_{\text {jet }}=3 \mathrm{~cm}$ or $\alpha_{\text {jet }}=20^{\circ}$, are only able to block the smallest aerosols. However, their combined effect under identical conditions is effective in blocking droplets up to $100 \mu \mathrm{m}$, which is considered to be the upper minimum diameter of virus-laden airborne aerosols (Wang et al., 2021). Further simultaneous increases in these parameters further increase the diameter of droplets are blocked. These numerical results quantitatively reflect our intuitive reasoning above, that simultaneous increases $y_{\mathrm{jet}}$ or $\alpha_{\mathrm{jet}}$ has a greater effect than the sum of each individual contribution.
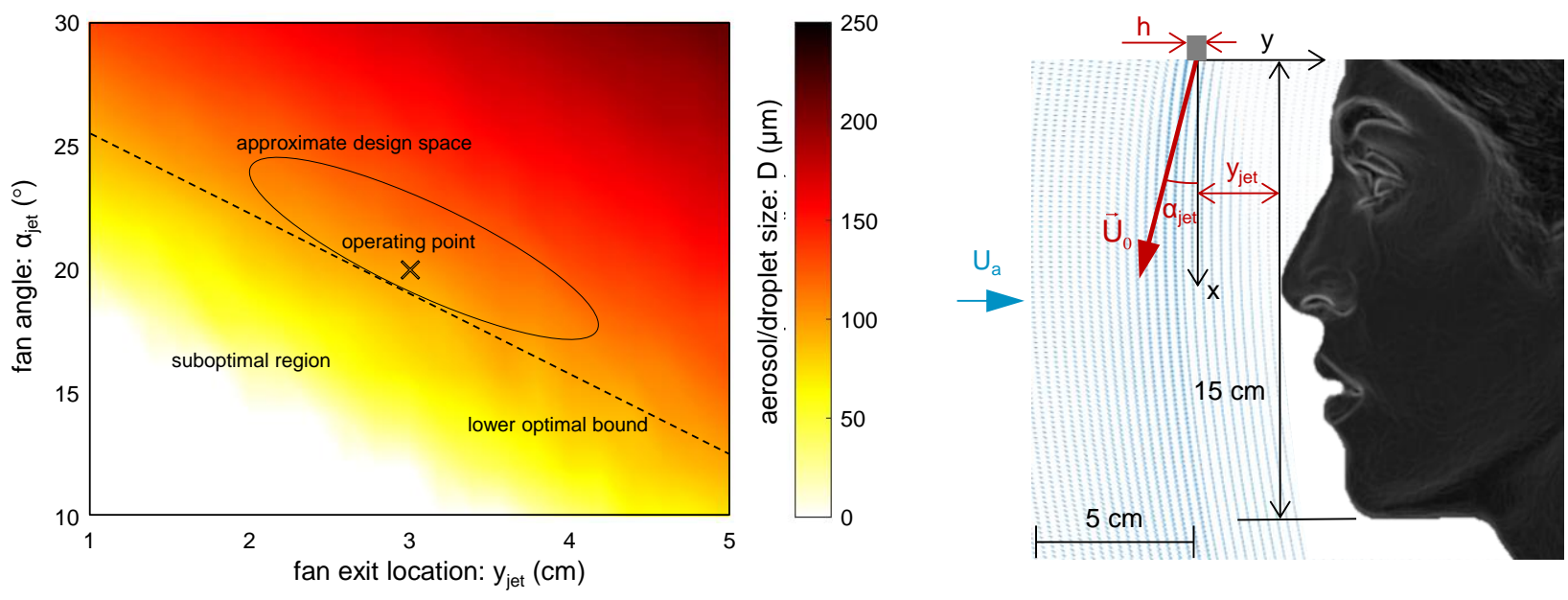

Figure 4. Left: Numerical simulations results showing the maximum airborne droplet diameter that the Air-Screen can block as a function of jet distance from face and angle relative to the vertical. Air-Screen parameters: $U_{0}=9 \mathrm{~m} / \mathrm{s}$, $h=5 \mathrm{~mm}$; crossflow $U_{a}=1.5 \mathrm{~m} / \mathrm{s}$; Right: schematic illustrating the varied jet parameters.

Although increases in the distance $y_{\text {jet }}$ effectively block larger aerosols and particles, there is a practically viable limit for which the fan can be comfortably and ergonomically incorporated into a cap. In addition, if $y_{\text {jet }}$ is too large, then the face is exposed to the possibility of virus-laden aerosols in yawed crosswinds that can obliquely enter the region between the fan and face. Regarding increases in $\alpha_{\text {jet }}$, there is also a practical limit determined by high levels of turbulent diffusion in the lower part of the jet which increases the possibility of virus-laden aerosol penetration in this region. Accordingly, we can define an "optimum design space" (shown in Figure 4) that maximizes the efficiency of the Air-Screen against large aerosols of $D \approx 100 \mu \mathrm{m}$ (Wang et al., 2021). Also indicated on the figure is the operating point of our prototype, the results of which are discussed in section 4 .

Visualization of droplets pathlines embedded in a crossflow velocity of $U_{a}=1.5 \mathrm{~m} / \mathrm{s}$, and using the acquired jet velocity field corresponding to $y_{\text {jet }}=3 \mathrm{~cm}$ and $\alpha_{\text {jet }}=20^{\circ}$, is shown in Figure 5. The boundary condition $\vec{u}_{\text {drop }}=\vec{U}_{a}$ along the left inflow boundary at $20 \mathrm{~cm}$ from the model was employed. The trajectories of three different droplet sizes are shown, namely $D=5 \mu \mathrm{m}, 100 \mu \mathrm{m}$ and $300 \mu \mathrm{m}$, both neglecting and including body forces. It can be seen that with no fan active (Figure $5 \mathrm{a}, \vec{F}_{b}=0$ ), all particles impact the face. Small particles of $5 \mu \mathrm{m}$ (Figure $5 \mathrm{~b}, \vec{F}_{b}=0$ ) are deflected to a relatively large distance from the face because, due to their low mass, they are easily accelerated (curved away). Further increases in diameter to $100 \mu \mathrm{m}$ (Figure $5 \mathrm{c}, \vec{F}_{b}=0$ ) show reduced effectiveness as the acceleration is diminished, but no penetration is observed. For $300 \mu \mathrm{m}$ droplets (Figure $5 \mathrm{~d}, \vec{F}_{b}=0$ ), even though droplets penetrate the jet, those approximately in line with the mouth can be sufficiently deflected away from the face. Figure 5e and Figure $5 \mathrm{f}$ illustrate that neglecting gravitational forces $\left(\vec{F}_{b} \neq 0\right)$ produces conservative estimates. 

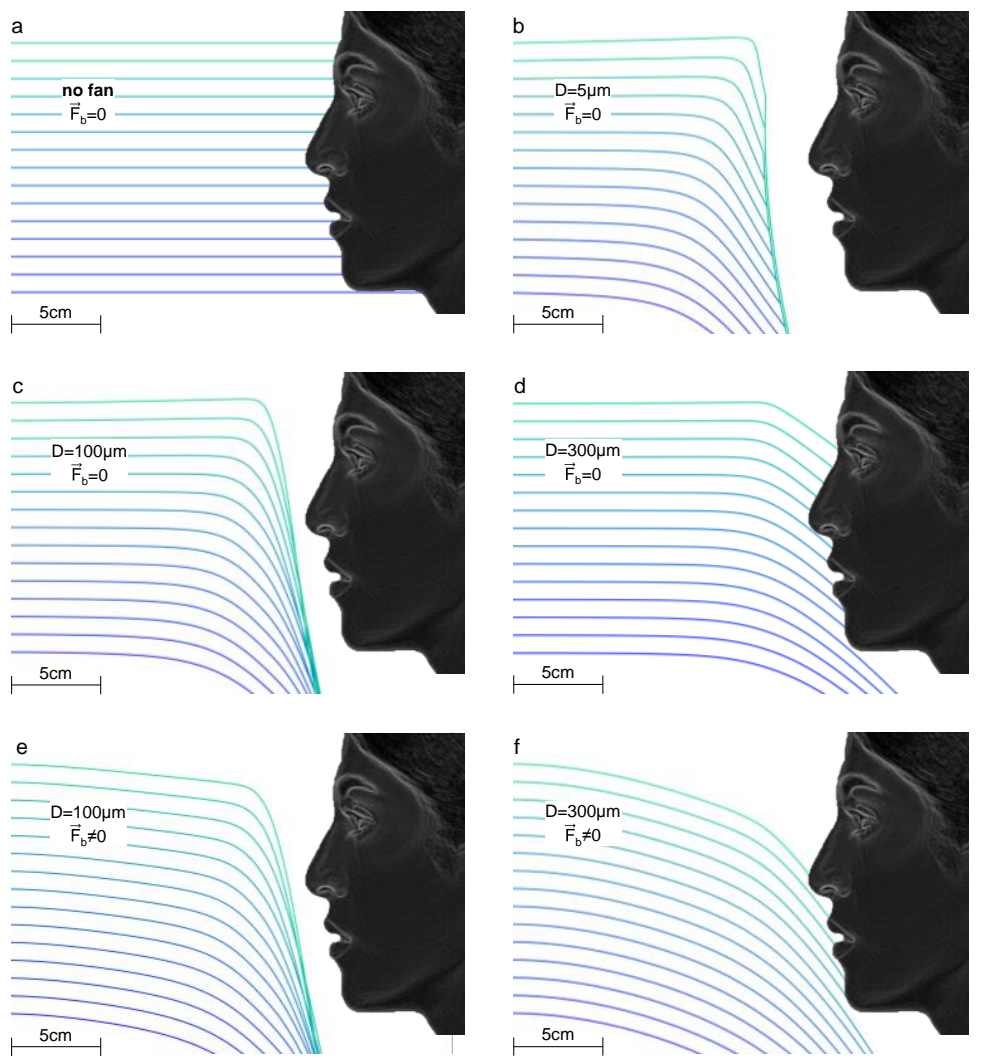

Figure 5. Numerical simulation of particles trajectories at a crossflow velocity of $U_{a}=1.5 \mathrm{~m} / \mathrm{s}$ and fan parameters: $U_{0}=9 \mathrm{~m} / \mathrm{s}, d=5 \mathrm{~mm}, y_{\text {jet }}=3 \mathrm{~cm}, \alpha_{\text {jet }}=20^{\circ}$ (a-d: gravitational forces neglected in simulations; e,f: gravitational forces included in simulations).
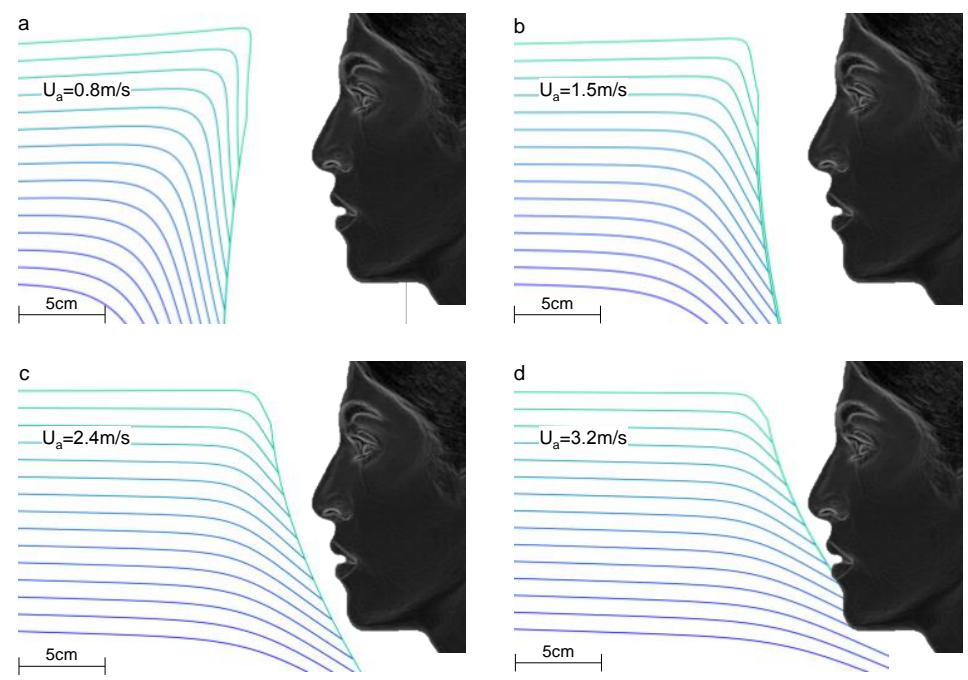

Figure 6. Numerical simulation of aerosol $(D=1.2 \mu \mathrm{m})$ trajectories of at various crossflow velocities. $U_{0}=9 \mathrm{~m} / \mathrm{s}$, $h=5 \mathrm{~mm}, y_{\mathrm{jet}}=3 \mathrm{~cm}$ and $\alpha_{\mathrm{jet}}=20^{\circ}$. Body forces are included in the simulations but have a negligible effect on $D=1.2 \mu \mathrm{m}$ aerosols. 
In our small aerosol experiments, presented below in section 4.1, we considered aerosols of $D=1.2 \mu \mathrm{m}$ under the conditions described above, where crossflow speeds $U_{a}$ of $0.8 \mathrm{~m} / \mathrm{s}, 1.5 \mathrm{~m} / \mathrm{s}, 2.4 \mathrm{~m} / \mathrm{s}$ and $3.2 \mathrm{~m} / \mathrm{s}$ were considered. This range was designed to cover speeds of slow walking or typical movements in an indoor environment to fast jogging. Aerosol pathline results of numerical simulations corresponding to these conditions are shown in Figure 6. These results indicate that blockage is effective up to $U_{a}=2.4 \mathrm{~m} / \mathrm{s}$, which is a typical jogging speed (5.4 miles/hour). The correspondence between these results and the experimental visualizations (Figure 12) is discussed further in section 4.1 .

The results presented above correspond to a single fan operating point where the fan momentum flux is $J_{0}=31 \mathrm{mN}$, based on our measurements presented in section 2.1. However, the fan power can be controlled, and as a result, the momentum flux or jet velocity distribution can be varied to accommodate different environmental conditions. Figure 7 illustrates the effect of the momentum flux on the minimum airborne droplet diameter necessary to penetrate through the fluidic barrier compared to the nominal operation regime for the parameters used in Figure 5 and Figure 6 . There is an almost linear relationship between the efficacy of the fluidic barrier and the momentum flux, and clearly, this motivates in favor of greater power input. However, this will impact noise and power consumption (battery capacity) which is proportional to $J^{1.5}$. Future research should aim to increase the system's efficiency by adapting the fan momentum flux, and hence input power, to the prevailing surrounding conditions.

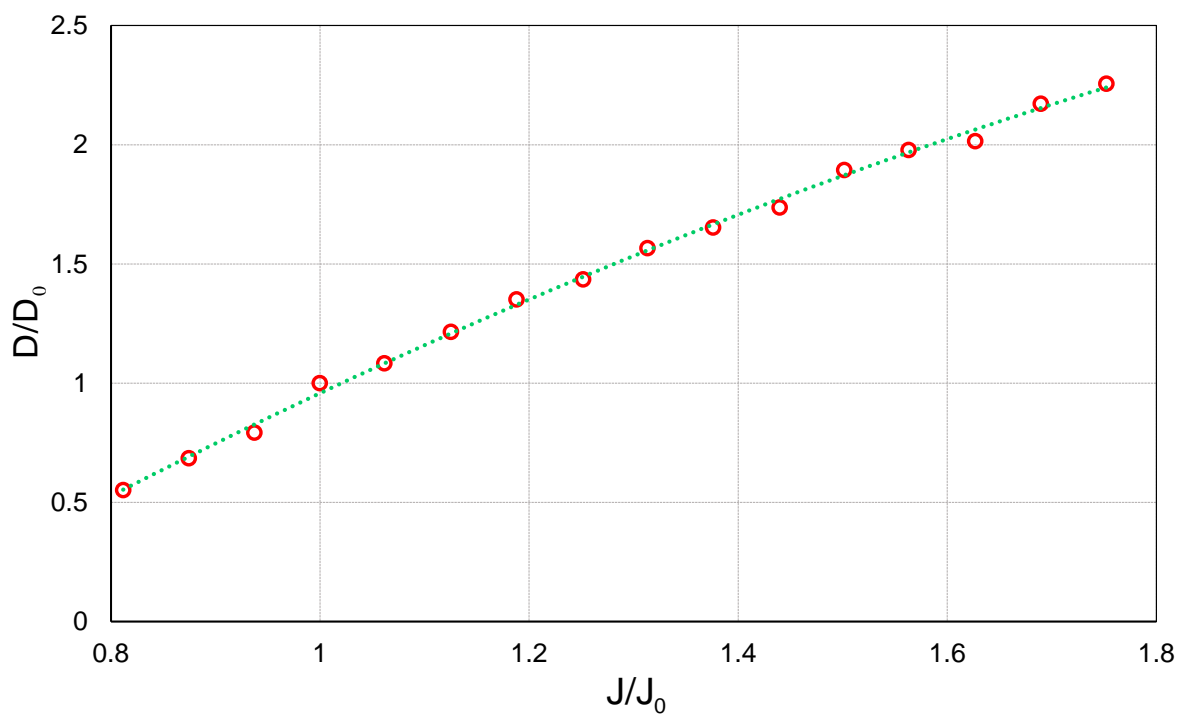

Figure 7. Numerical simulations results showing the effect of momentum flux on the minimal airborne droplet diameter necessary to penetrate through the fluidic barrier, referenced to $D_{0}=100 \mu \mathrm{m}$ and $J_{0}=31 \mathrm{mN}$. Fan and crosswind parameters: $y_{\text {jet }}=3 \mathrm{~cm}, \alpha_{\text {jet }}=20^{\circ}$ and $U_{a}=1.5 \mathrm{~m} / \mathrm{s}$.

The numerical simulation results presented above motivated strongly in favor of experimental verification presented in the next section. It should be noted, however, that the model is relatively simple, and it does not account for threedimensional (jet-edge) effects, yawed crosswinds, or reductions in airborne aerosol or droplet mass due to evaporation (Dbouk \& Drikakis, 2020a). The geometric (face) boundary condition is also not explicitly modeled. In future research, either these effects can be incorporated into the model or computational fluid dynamics methods (CFD), with appropriate turbulent models, can be employed.

\section{Experimental Configurations}

Following encouraging results from the numerical simulations, an experimental prototype was constructed (see Figure 1 ), with $y_{\text {jet }} \approx 3 \mathrm{~cm}$ and $\alpha_{\text {jet }} \approx 20^{\circ}$ (see the "operating point" indicated in Figure 4). Two sets of experiments were performed. The first set was qualitative and was used to visualize the effectiveness of the Air-Screen against small 
airborne aerosols $\left(D_{\text {drop }} \sim 10^{0} \mu \mathrm{m}\right)$, typically of those produced during oral communication or interactions during movement of the wearer. The second set was both qualitative and quantitative, where the effectiveness of the AirScreen was evaluated in the presence of large high-momentum droplets $\left(D_{\text {drop }} \sim 10^{2} \mu \mathrm{m}\right)$ typically produced during sneezing or coughing where social distancing is not enforced.

\subsection{Small Aerosol Setup}

Small aerosols were generated from DiEthyl-Hexyl-Sebacate (DEHS) (a colorless and odorless synthetic oil with a density of $\rho_{\text {DEHS }}=910 \mathrm{~kg} / \mathrm{m}^{3}$ ) using an ILATEC 40 seeder with six Laskin nozzles. This produced $D=1.2 \mu \mathrm{m}$ aerosols for a peak Q3 distribution according to the manufacturer's specification. The seeder was fed into a laminarization chamber via a flexible hose (see Figure 8, left) consisting of a baffle plate, three turbulence-reducing screens and a bell-mouth contraction leading to a $25 \mathrm{~mm}$ nozzle (Figure 8). The exit bell-mouth contraction velocity in the "top-hat" region was measured using a $1 \mathrm{~mm}$ diameter total head probe, and a jet velocity range $0.8 \mathrm{~m} / \mathrm{s} \leq V_{\text {jet }} \leq 3.2 \mathrm{~m} / \mathrm{s}$ was considered. In addition, the volumetric flow was monitored via Dwyer, $0-4$ liters $/$ minute, rotameter and controlled by both a pressure regulation valve and flow regulators of the seeding generator. The AirScreen was fitted on the mannequin (see Figure 8 ) and placed $30 \mathrm{~cm}$ from the nozzle outlet.

A Phantom v7.3 high-speed camera, mounted on the right side of the mannequin, was used to record video images at 300 frames per second. A $180 \mathrm{~W}$ UV diode cannon, mounted below the mannequin, was used to illuminate the aerosols in the vicinity of the mannequin. A black synthetic mat was mounted on the mannequin's left side to minimize reflections and create a sharp contrast between aerosols and the surrounding air (see Figure 8). Experiments performed without the Air-Screen active are referred to as "baseline" conditions.

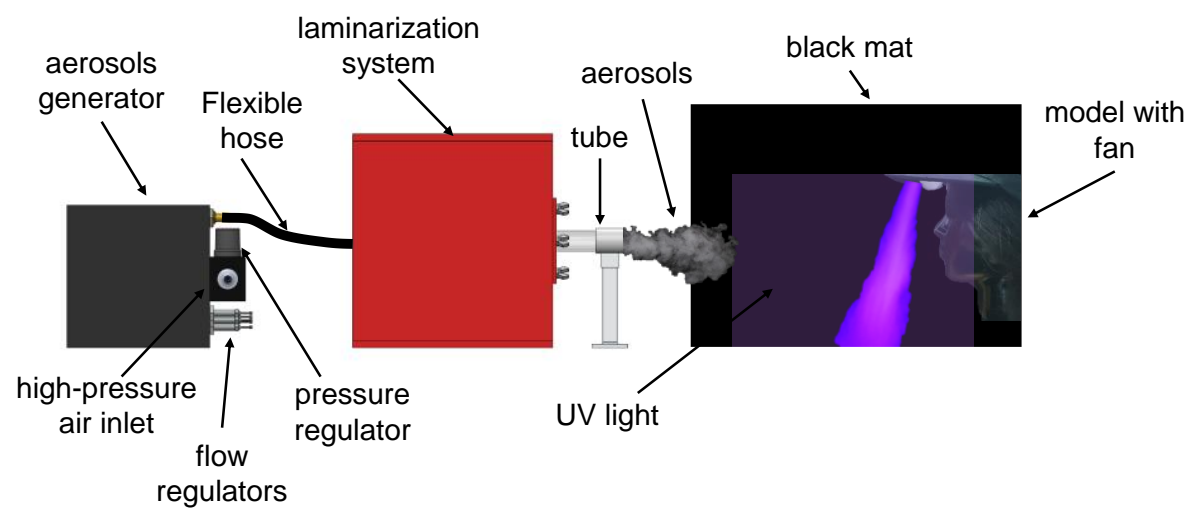

Figure 8. Schematic of the experimental configuration used to evaluate the Air-Screen against small aerosols.

\subsection{Large Droplet Setup}

Large droplets were generated utilizing a Paasche VL1007 hollow cone nozzle airbrush with a needle of size $0.7 \mathrm{~mm}$. The high-pressure inlet to the nozzle was controlled via a pressure regulating valve and produced an exit velocity of up to $50 \mathrm{~m} / \mathrm{s}$, measured at $1 \mathrm{~cm}$ from the nozzle exit. A syringe was used to feed the airbrush, and the water flow-rate was monitored at approximately 0.005 liters/minute. The airbrush was set at an angle of $10^{\circ}$ to the horizontal and located at $100 \mathrm{~cm}$ from the mannequin. A second Air-Screen fan was mounted $4 \mathrm{~cm}$ and $10 \mathrm{~cm}$ in front of and above the airbrush, respectively.

In this experimental setup, the airbrush was considered to be the "transmitter". Four separate sets of experiments were formed (a) baseline (no Air-Screen active); (b) the mannequin Air-Screen active; (c) the transmitter Air-Screen active; and (d) both Air-Screen fans active. Data were acquired using two independent methods, one qualitative and one quantitative. For the qualitative methods, a $1 \mathrm{~mm}$ wide laser sheet was generated using a $1.2 \mathrm{~W}, 532 \mathrm{~nm}$ green laser and laser sheet optics (see Figure 9). The high-speed Phantom v7.3 camera described above was used to detect droplets 
passing through the laser sheet at 1000 frames per second. A dedicated image-tracking algorithm was then used to identify the pathlines of droplets and render them onto a single image. A schematic of the full setup can be seen in Figure 9.

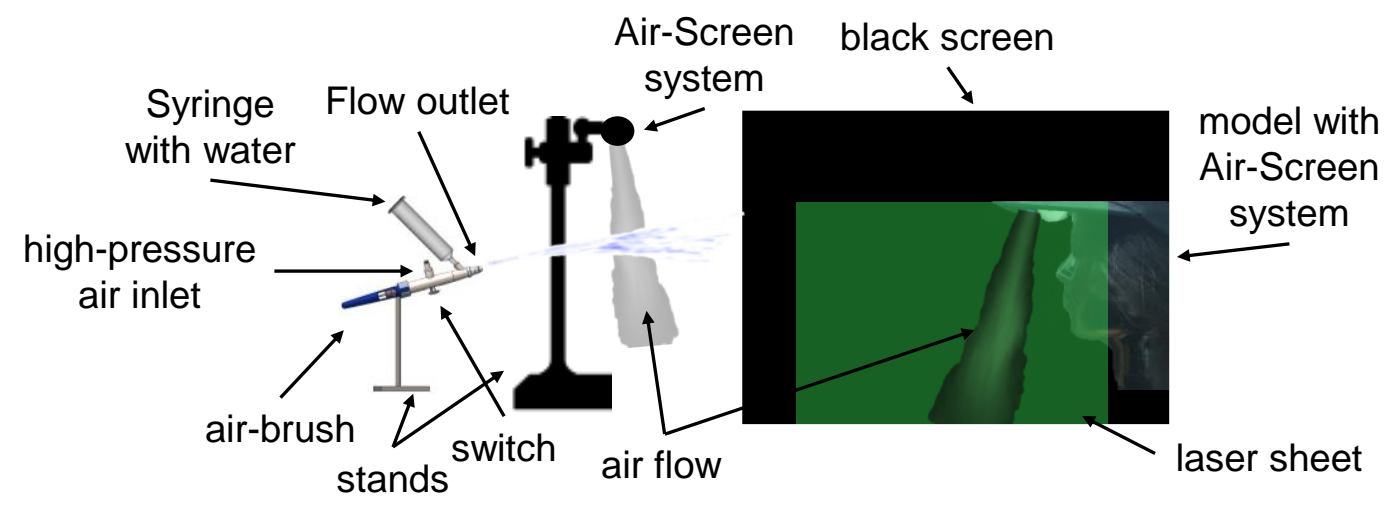

Figure 9. Schematic of the experimental configuration used to evaluate the Air-Screen against large droplets.

For the quantitative method, water-sensitive paper (WSP) sheets of dimensions $150 \times 120 \mathrm{~mm}$ were mounted on an aluminum frame $100 \mathrm{~cm}$ from the airbrush (instead of the mannequin), representing a frontal facial area. The airbrush was then operated for ten seconds for the four sets of experiments described above. For each experiment, a new piece of WSP was used. Following each experiment, a high-resolution image, $1200 \mathrm{dpi}$, of each WSP was acquired using a scanner and processed. Each image was segmented using Matlab $\odot$ image segmenter to produce an image mask. Examples of a scanned piece of WSP and its associated image mask are shown on the left and right in Figure 10. The masked data was obtained and analyzed through a dedicated algorithm that extracted the droplets' pixel size. By calculating the WPS size/pixel ratio, the droplets spread size could be evaluated. The actual droplet size was then determined using a WPS spreading to droplet size factor (Ghiani, Sassu, Piccirilli, Marcialis, \& Gambella, 2020). In so doing, the droplet diameter and population (or frequency) distribution could be determined. Due to the WSP and scanner limitation, the minimum decipherable droplet size was approximately $D=40 \mu \mathrm{m}$, characterizing the minimum range of large aerosol droplets.

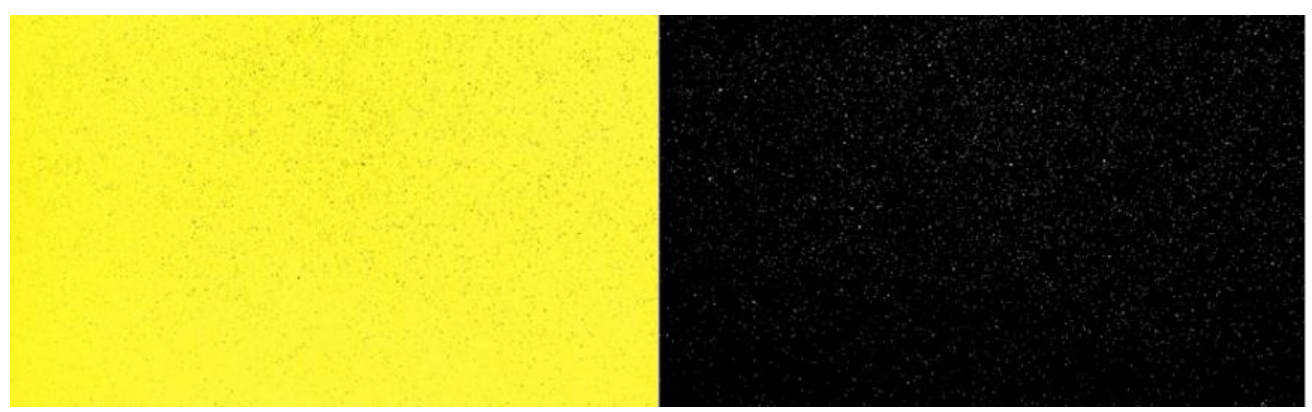

Figure 10. Example of a $150 \mathrm{~mm} \times 120 \mathrm{~mm}$ piece of WSP on the left and the results of the image segmenter used to produce an image mask of droplet size impacting the WSP on the right.

\section{Experimental Result and Discussion}

\subsection{Small Aerosols Experimental Results}

Initial experiments were designed to investigate the ability of the Air-Screen to shield against small size aerosols, $D \sim 10^{\circ} \mu \mathrm{m}$, as described in section 2.2. Figure 11 shows pairs of raw image pairs arbitrarily extracted from the highspeed camera recordings for baseline and activated Air-Screen cases (left and right). Four cases are shown where the 
jet velocity increased from $0.8 \mathrm{~m} / \mathrm{s}$ to $3.2 \mathrm{~m} / \mathrm{s}$. These cases correspond approximately to the numerical simulation results shown in Figure 6. It is important to note that these relatively high jet velocities exceed those that transpire during ordinary oral communication at these distances (Teager, 1980). These experiments also represent an individual working or walking or jogging in a field of aerosol-laden air. This can occur in environments containing small aerosols $(<5 \mu \mathrm{m})$, where pathogens predominate and can remain airborne in still air for long periods without sinking to the ground or evaporating (Fennelly, 2020).

In all baseline cases, the face is effectively smothered by the aerosol, where the amount of smothering increases with jet velocity. This is simply a consequence of the fact that more aerosol-laden air diluted with the ambient air. In contrast, a clear demarcation can be seen between the clean air and the aerosol-laden air when the Air-Screen is activated. This is an unambiguous effect of the Air-Screen. The only exception is $V_{\text {jet }}=3.2 \mathrm{~m} / \mathrm{s}$ where the aerosol appears to penetrate the bottom part of the facial area. This is only partially correct because aerosol-laden air is diverted around the sides of the mannequin, and these appear to be penetrating the screen. The smothering effect observed in the baseline cases demonstrates that even if a person wears a surgical mask, the aerosol can still be sucked in from around the mask due to the full facial coverage. Moreover, conventional masks provide no protection of the eyes where contamination due to small aerosols can occur (Chu et al., 2020). Clearly, the Air-Screen will provide even greater protection of the eyes due to the relatively high jet closer to the fan. It should also be appreciated that the main reason for wearing surgical or other facemasks is to minimize the chance of an infected individual infecting others; their ability to protect the wearer is unproven (Teesing et al., 2020). In contrast, these images clearly illustrate the ability of the Air-Screen to protect the wearer.
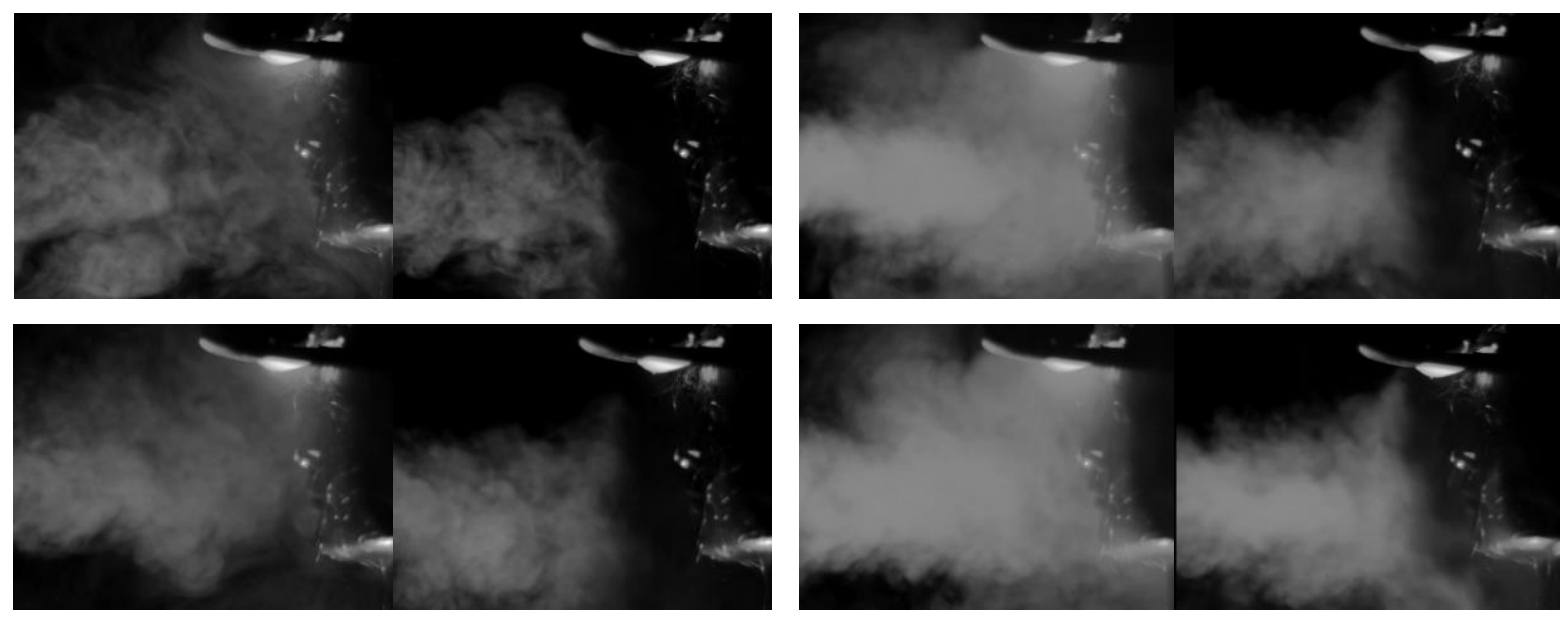

Figure 11. Arbitrary instantaneous images pairs of four aerosol experiments showing baseline (not activated) and activated Air-Screen cases (left and right). $V_{\text {jet }}=0.8 \mathrm{~m} / \mathrm{s}$ (top left); $1.6 \mathrm{~m} / \mathrm{s}$ (bottom left); $2.4 \mathrm{~m} / \mathrm{s}$ (top right); and 3.2 $\mathrm{m} / \mathrm{s}$ (bottom right).

An identical representation of these images is shown in Figure 12, where all images are averaged. These images show a clear contrast between the baseline and activated cases. The averaged images were processed, based on the brightness distribution of the captured data, using a segmentation code and based on the camera's optical sensor to evaluate the borders satisfying $3 \% \pm 2 \%$ of the aerosol's density. The low precision of the aerosol density borders is due to the changing brightness field each particle emits when exposed to focus a light source illuminating at partial of the full spectrum of the visible light. Nevertheless, this technique allows a more precise method to qualify the effectiveness of the Air-Screen against small aerosols. The images acquired show a strong resemblance to those acquired by numerical means (see Figure 6). The variance that exists between numerical and experimental results can be attributed to three main elements: (1) The crossflow introduced in the experiment was created via a round orifice in comparison to 2-D plane crossflow in the numerical calculations (2) The numerical calculation ignored 3D effects, and local turbulence (3) crossflow speed changed with the distance from the face, due to momentum conservation. Nevertheless, the experimental data acquired verify the numerical model suitability as an essential design tool for the Air-Screen. 

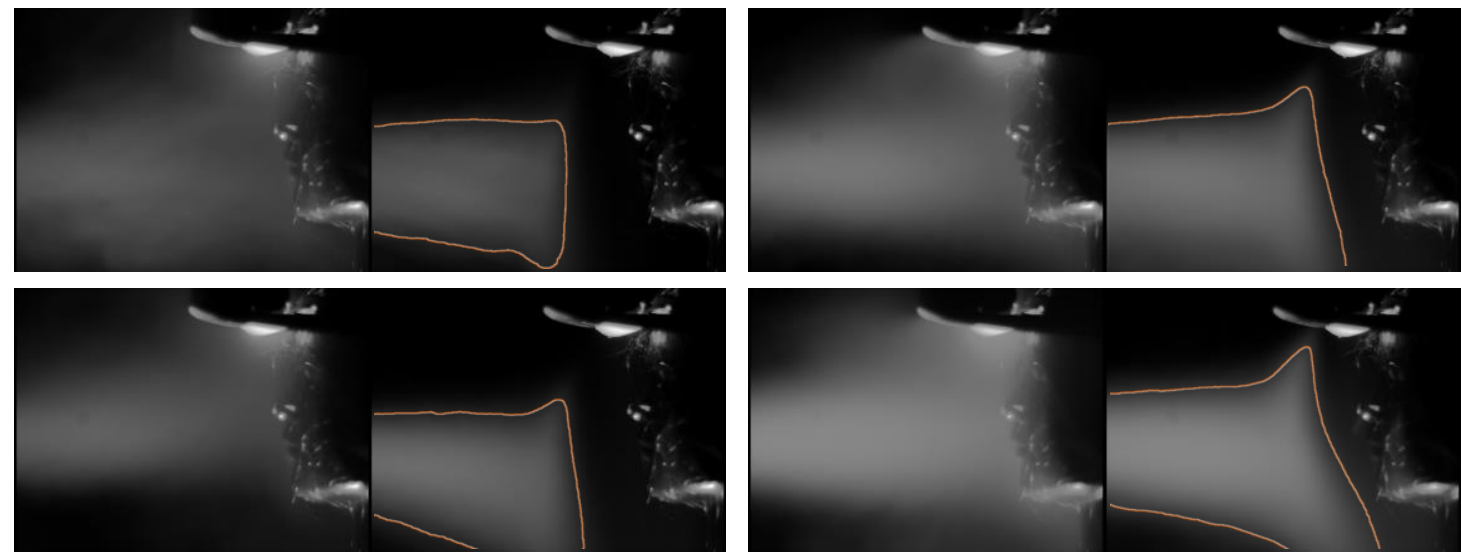

Figure 12. Averaged image pairs images for four experiments showing baseline and activated Air-Screen cases (left and right), where orange borders represent the $95 \%-99 \%$ demarcation limit: $V_{\text {jet }}=0.8 \mathrm{~m} / \mathrm{s}$ (top left); $1.6 \mathrm{~m} / \mathrm{s}$ (bottom left); $2.4 \mathrm{~m} / \mathrm{s}$ (top right); and $3.2 \mathrm{~m} / \mathrm{s}$ (bottom right).

\subsection{Large Droplets Experiments Results}

Following the small aerosol experiments, extensive qualitative and quantitative droplet transmission experiments were conducted. For the qualitative experiment, the droplets were recorded at 1000 frames/second, five times, over a onesecond interval at a time. Using a dedicated image process program, the pathlines of the droplets were processed and shown in Figure 13. Note that only droplets that pass through the $1 \mathrm{~mm}$ thick laser sheet are illuminated and recorded. The figure is arranged to show the baseline case (all Air-Screens off) and the other three combinations. It can be seen that when the mannequins' Air-Screen is activated, the number of water particles passing through the sheet diminishes. However, when the transmitter Air-Screen is activated, the number of particles is diminished virtually to zero. This is a clear demonstration that the Screen can protect others from an infected wearer. With both Screens active, the situation remains visually the same.

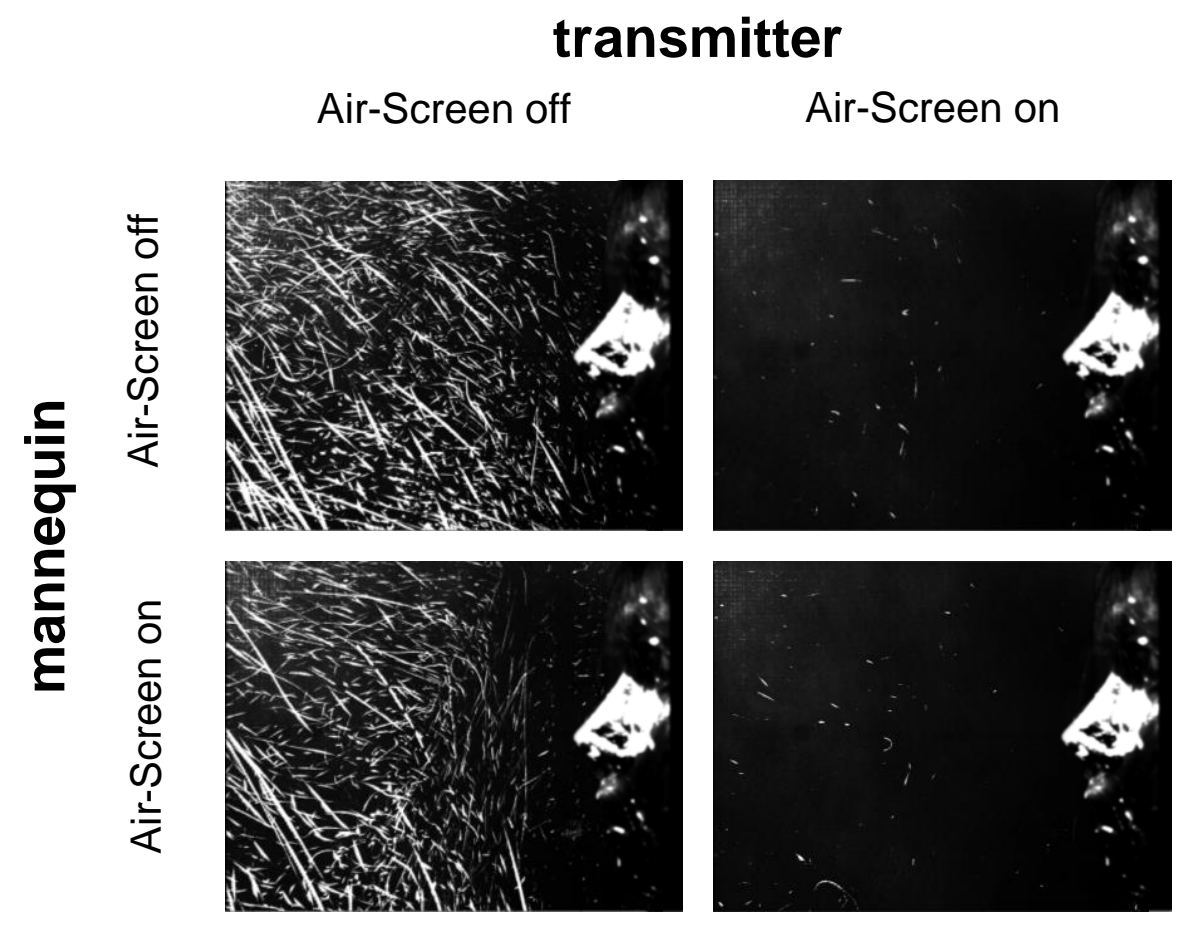

Figure 13. Partial droplets pathlines, passing through the laser sheet during one second, rendered onto four single images from recordings of 1000 frame/s. 
The observations made in the qualitative experiments are reflected in the quantitative WPS results. In Figure 14, an example of a single set of the WPS experiments is shown, out of 24 different experiments made with various droplet sizes. The black dots on the yellow WPS indicate where the water droplets have impacted the paper. Note that the actual aerosol size is smaller than the observed dot due to the spreading factor (Ghiani et al., 2020) explained in section 2. It should also be appreciated that the WSP is placed relatively low, corresponding to the position of the nose and mouth, where the Air-Screen jet velocity is relatively low. Despite this, the Air-Screen is remarkably effective when worn by the transmitter.

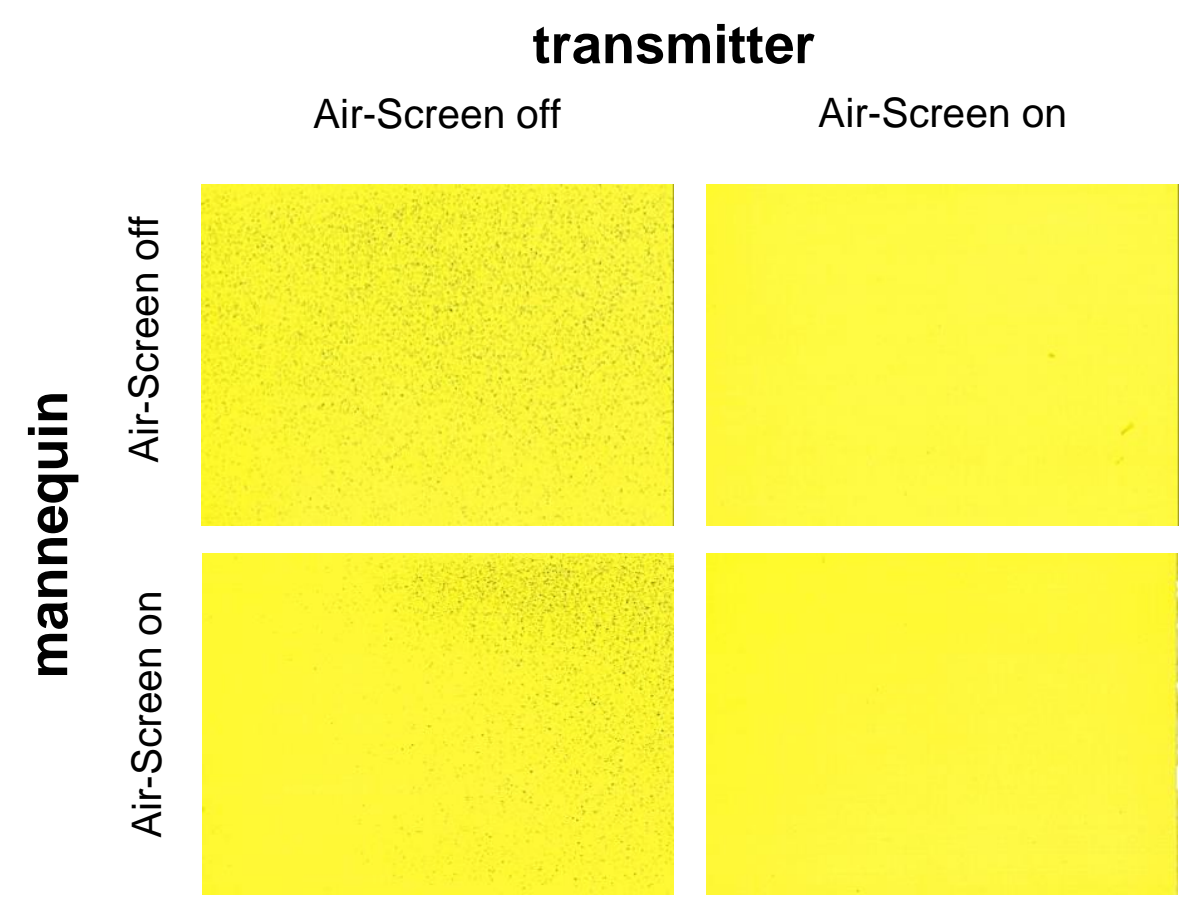

Figure 14. Scanned images of unprocessed WSP for a single set of experiments. The black dots reflect the impact of the water droplets on the paper.

This data was quantified using the image segmentation program to extract the droplet's size and apply the spreading corrections. The data gained is presented in the form of a histogram in Figure 15. The data were classified into two groups: (i) experiments with a droplet size of $D \gtrsim 150 \mu \mathrm{m}$, and (ii) experiments with a droplet size of $D \leqslant 150 \mu \mathrm{m}$.

This analysis shows several important aspects. When the mannequin Air-Screen is activated, it eliminates more than $62 \%$ of all the droplets from the size $D<150 \mu \mathrm{m}$. This shows the efficacy of the Air-Screen not only for effectively shielding against aerosols but also as an effective shield against relatively very large droplets. As anticipated, when the transmitter's Air-Screen is activated, it blocks more than 99\% of high momentum droplets issued at a distance of $100 \mathrm{~cm}$. When both Air-Screens are active, 99.8\% of the droplets are eliminated (see the inset in Figure 15). In fact, on average, only a single droplet, $D=330 \mu \mathrm{m}$, impacted the WSP out of ten of thousands of droplets aimed at the mannequin during the experiments. Hence, the key to success against even the largest droplets appears to be the AirScreen worm by a transmitter. The transmitter Screen diverts the larger droplets sufficiently downwards such that they are far less likely to impact the face at a similar height. 

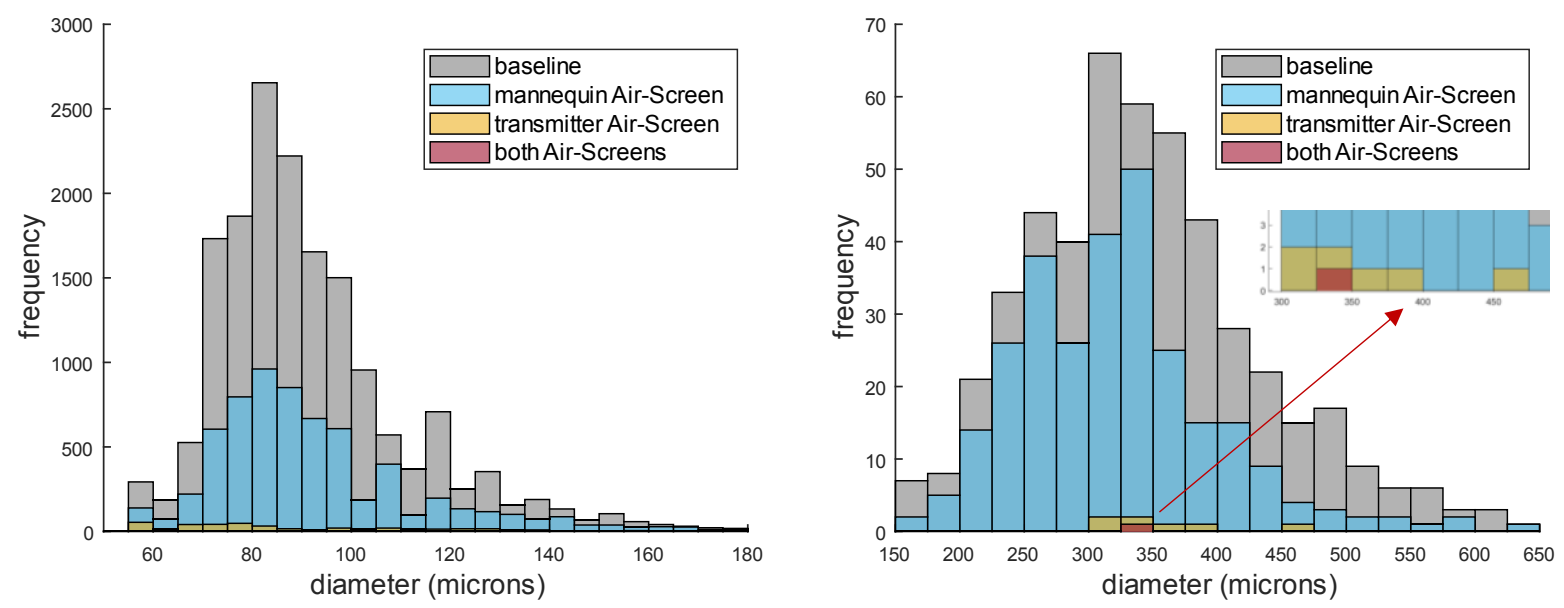

Figure 15. Histogram of the WSP data that was processed using an image mask to extract droplets' size and the corrections for spreading. (right) medium-large droplet experimental setup, $\tilde{D}=93 \mu \mathrm{m} \pm 24 \mu \mathrm{m}$. (left) very large droplet experimental setup, $\tilde{D}=273 \mu \mathrm{m} \pm 131 \mu \mathrm{m}$. (Inset: magnified area showing effects of transmitter and transmitter-mannequin Air-Screens)

To understand the implication of the experimental data, a more intuitive representation is shown in Figure 16. The model shown in the figure is based on a synthetic image generated by StyleGAN code (Karras et al., 2020) and corresponds to an archetypal face for illustrative purposes. The image represents the location of the WPS, illustrating a slightly larger morphological facial extent than average (Farkas et al., 2005). The figure exemplifies the distribution of the droplet penetration of the facial area with respect to the downstream direction as gained from the experiments with a droplet size of up to $200 \mu \mathrm{m}$. It is evident that for large droplets, the Air-Screen blows the droplets downward, thereby providing greater protection of the eyes and nostrils, as discussed in the previous subsection, due to the higher velocity jet closer to the fan. As can be inferred from the above results shown in Figure 13 to Figure 15, these images graphically illustrate the protection that the Air-Screen affords the target, particularly when worn by the transmitter. In conclusion, the Air-Screen provides excellent protection against high momentum droplets to the individual when worn by a transmitter and high protection when only used by the target.
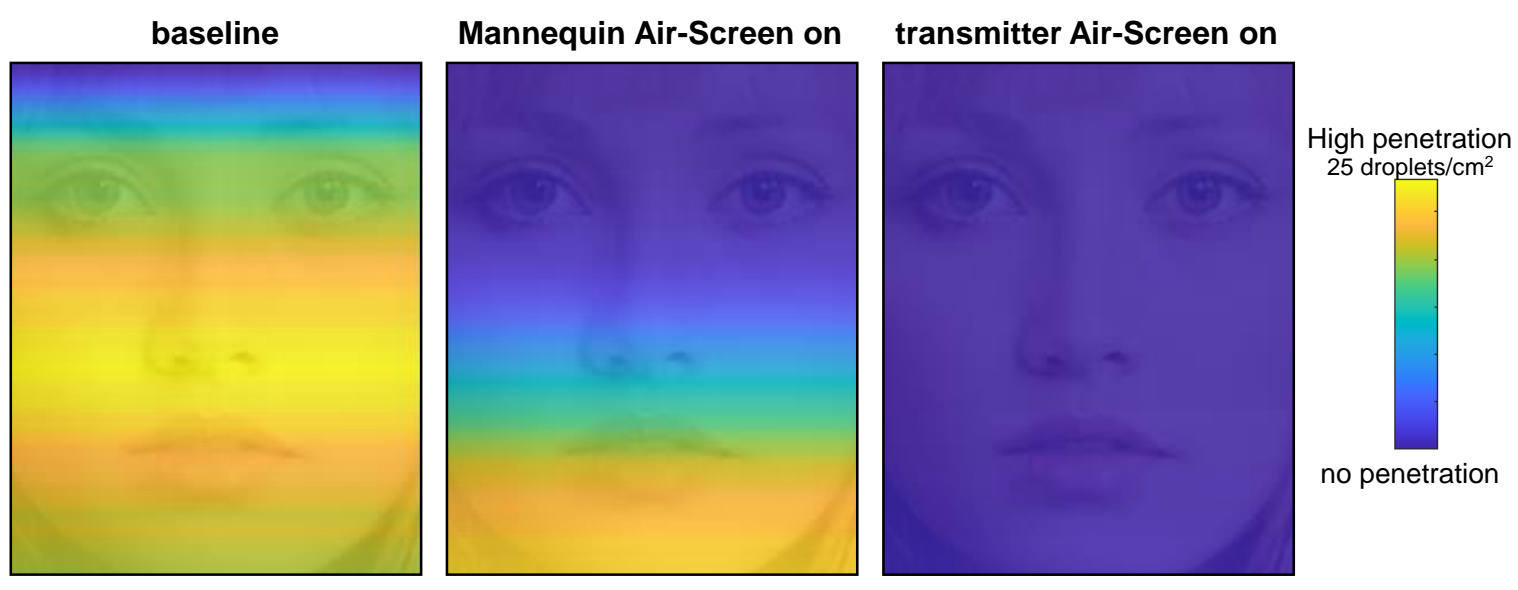

Figure 16. Average distribution of droplets penetration along face length as acquired from WSP experiments. Droplet size, $\tilde{D}=93 \mu \mathrm{m} \pm 24 \mu \mathrm{m}$ : left - baseline; middle -mannequin Air-Screen active; right - mannequin and transmitter Air-Screens active. 


\section{Conclusions \& Recommendations}

This study presented a theoretical basis for, and an experimental evaluation of, a fluidic facemask, called the AirScreen. Numerical simulations were performed under the assumption that the interaction between aerosol and droplet containing flows and the fluidic jet produces a weakly advected jet. Simulation results provided a relationship between effectiveness of the mask as a function of jet position and orientation. Furthermore, the results were used to determine a jet position and orientation "operating point" on which to base experiments.

Experiments performed using a mannequin clearly demonstrated that the Air-Screen effectively protects a wearer against airborne aerosols and droplets and, furthermore, protects others from an infected wearer. The experiments included both small-sized airborne aerosols $\left(D \sim 10^{0} \mu \mathrm{m}\right)$ and large droplets $\left(D \sim 10^{2} \mu \mathrm{m}\right)$ generated by sneezing or hard coughing.

For small aerosols, without an Air-Screen active, the face is smothered in airborne particles that can impact the eyes and even wearing a surgical mask does not eliminate the danger of inhaling the virus-laden aerosols. In contrast, the Air-Screen produced a clear demarcation between clean aerosol-free air and aerosol-laden air. For large droplets, the Air-Screen eliminated most of the droplets with a diameter of less than $150 \mu \mathrm{m}$ produced by a "transmitter". With the transmitter Air-Screen activated, 99\% of all droplets were eliminated, and when both Air-Screens, virtually all droplets were eliminated. The primary conclusion of this evaluation shows that the Air-Screen is remarkably effective in protecting the wearer and protecting others from an infected wearer.

Future studies can directly quantify the effectiveness of the Air-Screen and, in particular, compare it to surgical and other commercial facemasks using standard test methods. Alternately, a "breathing mannequin" can be employed where the inhaled aerosol concentration is directly quantified. In addition, an adaptable fan system can be introduced to regulate the jet momentum flux (input power) according to the relative ambient air velocity. In parallel, high fidelity CFD models, such as large eddy simulations (LES), can be used to simulate the full 3D problem and provide a solid foundation upon which to make design modifications and innovations.

Competing interests. None.

Declaration of Interests. The authors report no conflict of interest.

The data that support the findings of this study are available from Prof. David Greenblatt.

This research received no specific grant from any funding agency, commercial or not-for-profit sectors.

Ethical standards. The research meets all ethical guidelines, including adherence to the legal requirements of the study country.

Author contributions. Conceptualization: M.S., Funding acquisition M.S., Methodology: D.K; A.G; D.G., Data curation: D.K.; A.G.; D.G., Data Investigation D.K; Visualisation: D.K.; A.G., Software D.K, Supervision D.G., Writing - review \& editing: D.K.; D.G.. All authors approved the final submitted draft.

\section{List of abbreviations}

Centers for Disease Control and Prevention (CDC)

Corona virus air shield (CoVAS)

High-efficiency particulate air (HEPA)

Inlet guide vane (IGV)

DiEthyl-Hexyl-Sebacate (DEHS)

Water-sensitive paper (WSP)

Large eddy simulations (LES) 


\section{References}

Arumuru, V., Pasa, J., \& Samantaray, S. S. (2020). Experimental visualization of sneezing and efficacy of face masks and shields. Physics of Fluids, 32.

ASTM F1862 / F1862M-17. (2017). Standard Test Method for Resistance of Medical Face Masks to Penetration by Synthetic Blood (Horizontal Projection of Fixed Volume at a Known Velocity). West Conshohocken, PA, USA: ASTM International.

Bakhit, M., Krzyzaniak, N., Scott, A. M., Clark, J., Glasziou, P., \& Del Mar, C. (2021). Downsides of face masks and possible mitigation strategies: a systematic review and meta-analysis. BMJ Open, 11, e044364.

Bhagat, R. K., Wykes, M. S. D., Dalziel, S. B., \& Linden, P. F. (2020). Effects of ventilation on the indoor spread of COVID-19. Journal of Fluid Mechanics, 903.

Bourouiba, L., Dehandschoewercker, E., \& Bush, J. W. M. (2014). Violent expiratory events: On coughing and sneezing. Journal of Fluid Mechanics, 745, 537-563.

Brown, P. P., \& Lawler, D. F. (2003). Sphere Drag and Settling Velocity Revisited. Journal of Environmental Engineering, 129, 222-231.

BS EN 14683. (2019). Medical face masks. Requirements and test methods. In BSI Standards.

Cárdenas, C., Suntz, R., Denev, J. A., \& Bockhorn, H. (2007). Two-dimensional estimation of Reynolds-fluxes and stresses in a Jet-in-Crossflow arrangement by simultaneous 2D-LIF and PIV. Applied Physics B: Lasers and Optics, 88, 581-591.

CDC. (2021, May). Science Brief: Community Use of Cloth Masks to Control the Spread of SARS-CoV-2. Retrieved June 11, 2021, from CDC website: https://www.cdc.gov/coronavirus/2019-ncov/science/sciencebriefs/masking-science-sars-cov2.html

Chu, D. K., Akl, E. A., Duda, S., Solo, K., Yaacoub, S., Schünemann, H. J., ... Urgent, C.-S. (2020). Physical distancing, face masks, and eye protection to prevent person-to-person transmission of SARS-CoV-2 and COVID-19 : a systematic review and meta-analysis. Lancet, 395, 1973-1987.

Contal, P., Vendel, J., Leclerc, D., Renaudin, V., Penicot, P., \& Thomas, D. (1999). Modelling pressure drop in hepa filters during dynamic filtration. Journal of Aerosol Science, 30, 235-246.

Dang, T. Q., \& Bushnell, P. R. (2009). Aerodynamics of cross-flow fans and their application to aircraft propulsion and flow control. Progress in Aerospace Sciences, 45, 1-29.

Dbouk, T., \& Drikakis, D. (2020a). On coughing and airborne droplet transmission to humans. Physics of Fluids, 32.

Dbouk, T., \& Drikakis, D. (2020b). On respiratory droplets and face masks. Physics of Fluids, 32.

Farkas, L. G., Katic, M. J., Forrest, C. R., Alt, K. W., Bagič, I., Baltadjiev, G., .. Yahia, E. (2005). International anthropometric study of facial morphology in various ethnic groups/races. Journal of Craniofacial Surgery, 16, 615-646.

Fennelly, K. P. (2020). Particle sizes of infectious aerosols: implications for infection control. The Lancet Respiratory Medicine, 8, 914-924.

Frank, D., \& Linden, P. F. (2014). The effectiveness of an air curtain in the doorway of a ventilated building. Journal of Fluid Mechanics, 756, 130-164.

Ghiani, L., Sassu, A., Piccirilli, D., Marcialis, G. L., \& Gambella, F. (2020). Development of a Matlab Code for the Evaluation of Spray Distribution with Water-Sensitive Paper. In Lecture Notes in Civil Engineering (Vol. 67, pp. 845-853). Springer.

Gutmark, E., \& Wygnanski, I. (1976). The planar turbulent jet. Journal of Fluid Mechanics, 73, 465-495.

Haniu, H., \& Ramaprian, B. R. (1989). Studies on two-dimensiooal curved nonbuoyant jets in cross flow. Journal of Fluids Engineering, Transactions of the ASME, 111, 78-86.

Huang, J. F., Davidson, M. J., \& Nokes, R. I. (2005). Two-dimensional and line jets in a weak cross-flow. Journal of Hydraulic Research, 43, 390-398.

Kamotani, Y., \& Greberf, I. (1972). Experiments on a Turbulent Jet in a Cross Flow. AIAA Journal, 10, 1425-1429.

Karras, T., Laine, S., Aittala, M., Hellsten, J., Lehtinen, J., \& Aila, T. (2020). Analyzing and improving the image quality of stylegan. Proceedings of the IEEE Computer Society Conference on Computer Vision and Pattern Recognition, 8107-8116.

Kincaid, D., \& Cheney, W. (2002). Numerical Analysis: Mathematics of Scientific Computing. In american mathematical society ( 3 rd ed.).

Kisielinski, K., Giboni, P., Prescher, A., Klosterhalfen, B., Graessel, D., Funken, S., ... Hirsch, O. (2021). Is a Mask That Covers the Mouth and Nose Free from Undesirable Side Effects in Everyday Use and Free of Potential Hazards? International Journal of Environmental Research and Public Health 2021, Vol. 18, Page 4344, 18, 4344.

Kumar, A., Kasloff, S. B., Leung, A., Cutts, T., Strong, J. E., Hills, K., ... Krishnan, J. (2020). N95 mask 
decontamination using standard hospital sterilization technologies. MedRxiv.

Laine, C., Goodman, S. N., \& Guallar, E. (2020). The Role of Masks in Mitigating the SARS-CoV-2 Pandemic: Another Piece of the Puzzle. Annals of Internal Medicine, 18-20.

Proudman, I., \& Pearson, J. R. A. (1957). Expansions at small Reynolds numbers for the flow past a sphere and a circular cylinder. Journal of Fluid Mechanics, 2, 237-262.

Sakharov, A. S., \& Zhukov, K. (2020). Study of Air Curtain in Context of Individual Protection from Exposure to Coronavirus (SARS-CoV-2) Contained in Cough-Generated Fluid Particles. ArXiv, 2, 340-351.

Sarkar, A., Xu, F., \& Lee, S. (2019). Human saliva and model saliva at bulk to adsorbed phases - similarities and differences. Advances in Colloid and Interface Science, 273, 102034.

Scharfman, B. E., Techet, A. H., Bush, J. W. M., \& Bourouiba, L. (2016). Visualization of sneeze ejecta: steps of fluid fragmentation leading to respiratory droplets. Experiments in Fluids, 57, 1-9.

Schlichting, H., \& Gersten, K. (2016). Boundary-Layer Theory (9th ed.). Springer Berlin Heidelberg.

Teager, H. M. (1980). Some Observations on Oral Air Flow During Phonation. IEEE Transactions on Acoustics, Speech, and Signal Processing, 28, 599-601.

Teesing, G. R., Straten, B. Van, Man, P. De, \& Horeman-franse, T. (2020). Is there an adequate alternative to commercially manufactured face masks? A comparison of various materials and forms. Journal of Hospital Infection, 106, 246-253.

Thompson, C. A. (1965). Hot-wire measurements on a plane turbulent jet. Journal of Applied Mechanics, Transactions ASME, 721-734.

Verma, S., Dhanak, M., \& Frankenfield, J. (2020). Visualizing the effectiveness of face masks in obstructing respiratory jets. Physics of Fluids, 32 .

Wang, C. C., Prather, K. A., Sznitman, J., Jimenez, J. L., Lakdawala, S. S., Tufekci, Z., \& Marr, L. C. (2021). Airborne transmission of respiratory viruses. Science, 373, eabd9149.

Zhang, W., Deng, S., Wang, Y., \& Lin, Z. (2020). Modeling the surface filtration pressure drop of PTFE HEPA filter media for low load applications. Building and Environment, 177, 106905.

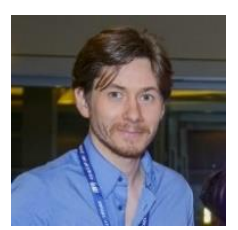

D. Keisar is Ph.D. student in the Grand Technion Energy Program. He received his B.Sc. degree in Mechanical Engineering and M.Sc. degree in Energy Engineering from the Technion, Israel. In addition, at the age of 18 he received his B.A. degree in Economics and management from the Open University, Israel.

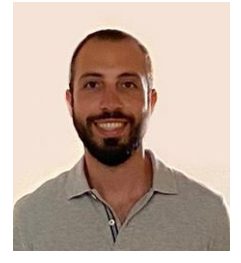

A. Garzozi is a Ph.D. student at the Technion's Grand Technion Energy Program (GTEP) and a research member of the Flow Control Laboratory group. He completed B.Sc degree in Mechanical Engineering and M.Sc. in Energy Engineering at the Technion Israel Institute of Technology.

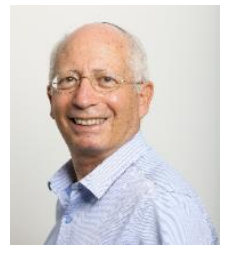

Prof. Shoham has B.Sc. in Aeronautical Engineering, served as an engineer in Israel Aircraft Industry, and then gained M.Sc. and D.Sc. in Mechanical Engineering from the Technion-Israel Institute of Technology, where he has been teaching for the past nearly 30 years, and is currently an endowed chair professor. International member of USA-National Academy of Engineering, Professor Shoham is the recipient of more than dozen awards, including: the Thomas A. Edison Patent Award. Fellow of IEEE and ASME, Prof. Shoham was formerly the head of the Robotic Laboratory of the Department of Mechanical Engineering at Columbia University, NY. he holds more than 50 patents, has published over 200 technical papers and is the author and co-author of three books. 


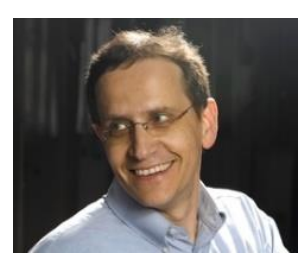

Prof. Greenblatt obtained BSc and MSc. degrees at the University of the Witwatersrand, South Africa and served as an engineer at the CSIR. He obtained his PhD at Tel Aviv University and conducted post-doctoral research at the Illinois Institute of Technology, NASA Langley Research Center and the Technical University of Berlin. He has authored or co-authored more than 170 technical publications, nine book chapters and ten patents. He is presently a Professor at the Technion's Faculty of Mechanical Engineering, Head of the Flow Control Laboratory and Head of The Research Center for Energy Engineering and Environmental Preservation. He is an Associate Fellow of the American Institute of Aeronautics and Astronautics. 